\title{
Silicon Nitride Overlays Deposited on Optical Fibers with RF PECVD Method for Sensing Applications: Overlay Uniformity Aspects
}

\author{
B. Michalak ${ }^{a}$, M. KobA ${ }^{b}$ And M. Śmietana ${ }^{a, *}$ \\ ${ }^{a}$ Institute of Microelectronics and Optoelectronics, Warsaw University of Technology, \\ Koszykowa 75, 00-662 Warsaw, Poland \\ ${ }^{b}$ National Institute of Telecommunications, Szachowa 1, 04-894 Warsaw, Poland
}

(Received May 12, 2014; in final form April 3, 2015)

\begin{abstract}
This work discusses uniformity of overlays deposited with radio frequency plasma enhanced chemical vapor deposition method on a core of partly uncoated multimode polymer-clad silica optical fiber. Although the method provides uniform films on flat surfaces, uniformity of deposition on optical fibers suspended in plasma is questionable. In this work we investigate optical-fiber-based structures coated with high-refractive-index $\left(n_{D}>2.3\right)$ silicon nitride $\left(\mathrm{SiN}_{x}\right)$ where lossy mode resonance phenomenon appears. Experimental studies, where the fiber is rotated by $90^{\circ}$ and $180^{\circ}$, are compared to those where the sample has not been rotated between the depositions. Deposition process time for all samples has been adjusted in order to obtain the same thickness of overlays on all the fibers. The experimental data has been supported by numerical simulations. The experiment has shown that the rotation modifies transmission of the $\mathrm{SiN}_{x}$-coated fiber structure, as well as its response to external refractive index. As an effect of rotation we observed shift of the resonance appearing at about $\lambda=600 \mathrm{~nm}$ towards shorter wavelengths and reduction in amount of the resonances. However, the resonances appearing in transmission spectrum for sample with no rotation show the highest refractive index sensitivity reaching about $690 \mathrm{~nm}$ per refractive index unit.
\end{abstract}

DOI: $10.12693 /$ APhysPolA.127.1587

PACS: 07.07.Df, 42.70.Ce, 42.79.Gn, 81.20.Fw

\section{Introduction}

There is a widespread interest in sensing devices based on optical fibers, especially optical fiber refractometers where detection system can be simplified to light intensity-based measurement [1, 2]. A segment of a fiber core exposed to external media only on tenths of $\mathrm{mm}$ length is one of the simplest sensing structures [3]. By applying an overlay on the exposed core, the overlay can absorb or bind certain stimulant resulting in variation of thickness and optical properties of the overlay, as well as its external medium. Formation of the overlay induces variation in propagation condition in the fiber [4]. High refractive index (high- $n$ ) overlays (typically $n_{D}>1.47$ ) are now widely studied due to their significant impact on the propagation in the fiber [5]. Relatively simple and repeatable overlay deposition methods with high flexibility in determination of overlay properties resulting in determination of sensing structure functionality are highly desired. Silicon nitride $\left(\mathrm{SiN}_{x}\right)$ offers high $n$ comparing to the one of fiber cladding. The films can be deposited with radio frequency plasma enhanced chemical vapor deposition (RF PECVD) method capable for easy and precise tuning of $n$ and thickness of the films [6]. These properties are crucial for obtaining high sensitivity of the fiberbased device. An effect obtained by coating a multimode

* corresponding author; e-mail: msmietan@elka.pw.edu.pl optical fiber core with thin and high- $n$ overlay is often called lossy mode resonance (LMR) $[7,8]$. External refractive index (RI) sensors based on LMR effect are of high interest due to their simplicity and high sensitivity $[2,9]$.

The RF PECVD setups are typically designed for film depositions on flat substrates and coating uniformity of cylindrical structure such as optical fiber is questionable. In this work we discuss uniformity of overlays deposited with the method on core of partly uncoated multimode polymer-clad silica (PCS) optical fiber. The deposition effect for the structure surrounded by different RI is investigated experimentally and numerically.

\section{Experimental details}

\subsection{Sample preparation}

All samples (150 mm in length) were based on PCS optical fiber with fused silica core $(\varnothing=400 \mu \mathrm{m})$. The center segment of the fiber has been chemically exposed to methylene chloride and then the cladding has been mechanically removed on length of $25 \mathrm{~mm}$.

\subsection{SiN $N_{x}$ overlay deposition}

The $\operatorname{SiN}_{x}$ has been deposited using Oxford Plasmalab 80 Plus system with parameters given in Table. A sample holder used for suspension of the structure in the RF PECVD reactor is schematically shown in Fig. 1. 
TABLE

RF PECVD process parameters of $\mathrm{SiN}_{x}$.

\begin{tabular}{c|c|c|c|c|c}
\hline $\begin{array}{c}\mathrm{SiH}_{4} \text { flow } \\
{[\mathrm{sccm}]}\end{array}$ & $\begin{array}{c}\mathrm{NH}_{3} \text { flow } \\
{[\mathrm{sccm}]}\end{array}$ & $\begin{array}{c}\mathrm{P} \\
{[\mathrm{W}]}\end{array}$ & $\begin{array}{c}\mathrm{P} \\
{[\mathrm{Torr}]}\end{array}$ & $\begin{array}{c}\mathrm{T} \\
{\left[{ }^{\circ} \mathrm{C}\right]}\end{array}$ & $\begin{array}{c}\mathrm{h} \\
{[\mathrm{mm}]}\end{array}$ \\
\hline 500 & 50 & 50 & 1.2 & 200 & 8.45
\end{tabular}

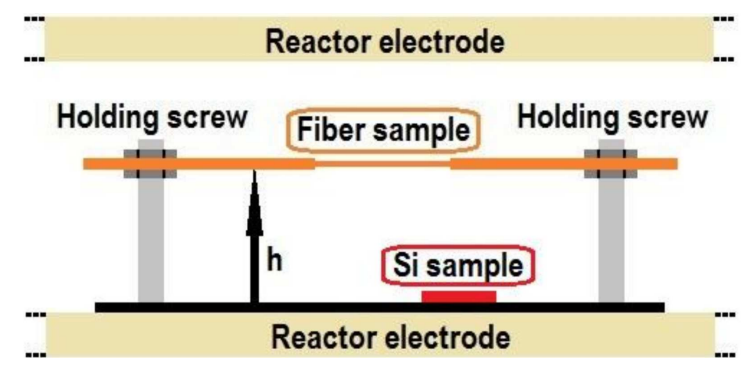

Fig. 1. Scheme of fiber holder in the PF PECVD reactor with adjustable sample height $(h)$.

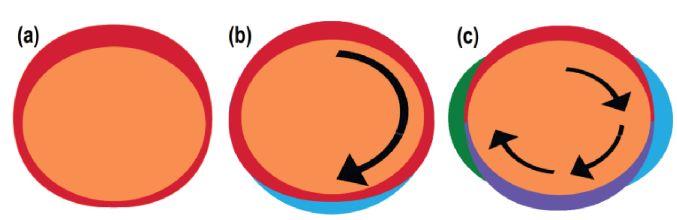

Fig. 2. Predicted effect of sample rotation, where (a) shows overlay deposition with no rotation, (b) with one $180^{\circ}$ rotation, and (c) with three $90^{\circ}$ rotations.

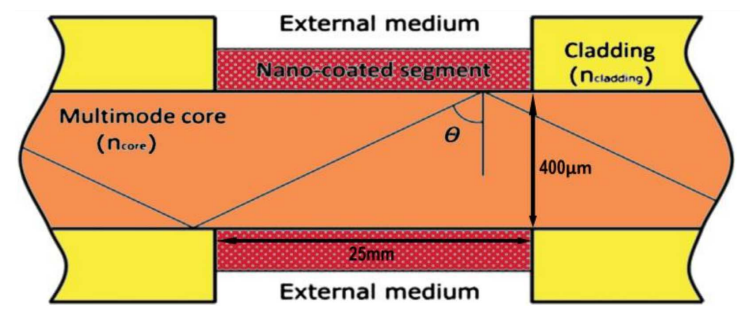

Fig. 3. Cross-section of the investigated fiber structure.

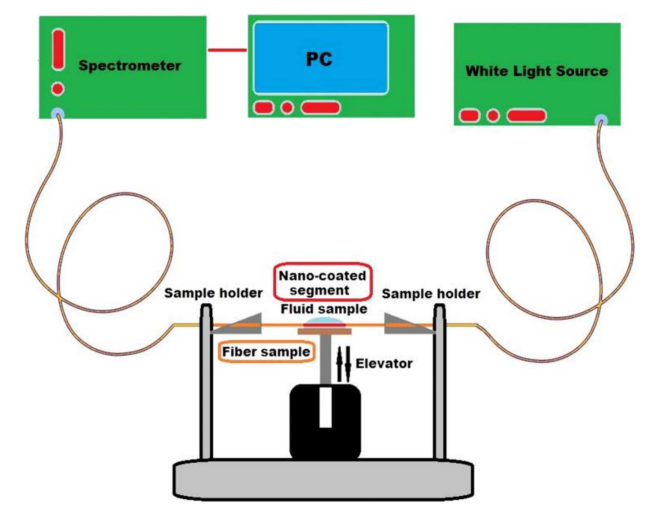

Fig. 4. Scheme of the experimental setup applied for external RI measurements.
Deposition time has been adjusted to obtain the same thickness of overlay on each sample despite of rotation. It was one $3 \mathrm{~min}$ long process for no rotation, two $1 \mathrm{~min}$ and $25 \mathrm{~s}$ long processes for $180^{\circ}$ rotation, and four $40 \mathrm{~s}$ long processes for $90^{\circ}$ rotation. Predicted effect of sample rotation on overlay thickness uniformity is shown in Fig. 2 where each color represents extended thickness due to fiber position during deposition. The obtained fiber structure is schematically shown in Fig. 3.

\subsection{Measurements of film properties}

Film thickness and its optical constants, i.e., $n$ and extinction coefficient $(k)$ were investigated on reference Si samples with Horiba Jobin-Yvon UVISEL spectroscopic ellipsometer in the wavelength range of 260 to $830 \mathrm{~nm}$. The samples were placed on the holder surface during the deposition process (Fig. 1). Ellipsometric measurements were fitted with DeltaPsi2 software using single layer Tauc-Lorenc model of $\operatorname{SiN}_{x}$.

\subsection{External RI measurements}

After the deposition the samples' end-faces were mechanically polished and cleaned chemically. Next, they were installed in the measurement setup (Fig. 4). Measurements were performed by the use of Ocean Optics USB4000 Spectrometer and Ocean Optics HL-2000, as a spectrum analyzer and a light source, respectively. Spectral response of the sensing element has been analyzed using Spectra Suite software for the coated segment immersed in liquids with $n_{D}$ of 1.3330 to 1.4534 . The $n_{D}$ of the liquid has been measured using Rudolph's Digital J57 refractometer. Obtained spectra were normalized with data for a reference sample with no overlay measured in air.

\subsection{Numerical simulations}

Simulation of the phenomena taking place in the device was performed using Matlab software supported with a code based on theoretical elaborations reported elsewhere [9]. As optical properties of the overlays we assumed those measured with spectroscopic ellipsometry. Thickness of the overlay was set up to $140 \mathrm{~nm}$.

\section{Results and discussion}

Thicknesses of the obtained films on reference samples are 57.8, 56.2 (thickness for each rotation 26.9, $27.0 \mathrm{~nm}$ ), and $53.1 \mathrm{~nm}$ (thickness for each rotation 13.2, 12.6, $13.3,14.4 \mathrm{~nm}$ ) for no rotation, $180^{\circ}$ and $90^{\circ}$ rotation, respectively. It can be seen that the deposition processes are highly repeatable. The change of the thickness for each process is due to different sum of deposition time, which was primarily adjusted to prevent the effect of nonlinear start of the deposition. The average deposition rate is about $19.3 \mathrm{~nm} / \mathrm{min}$ and at initial stage of film growth its kinetics is slightly higher. Optical properties of the films deposited on reference samples are shown in Fig. 5. 


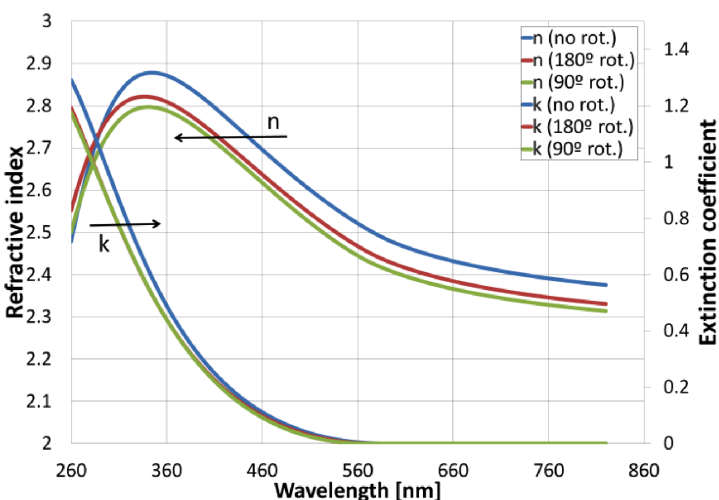

Fig. 5. Dispersion curves of refractive index $(n)$ and extinction coefficient $(k)$ for the $\operatorname{SiN}_{x}$ films deposited on the reference Si wafers. The data correspond to processes where fiber samples experienced rotation.

The properties increase with film thickness and this effect has been discussed in detail elsewhere [5]. Normalized transmitted power for obtained samples with different rotation is shown in Fig. 6 . It can be seen that the rotation causes one of the resonances to disappear, thus the remaining resonance becomes smoother and slightly narrower. It can be also observed that small variations of thickness for different deposition conditions cause shift of the resonance wavelength towards smaller values.

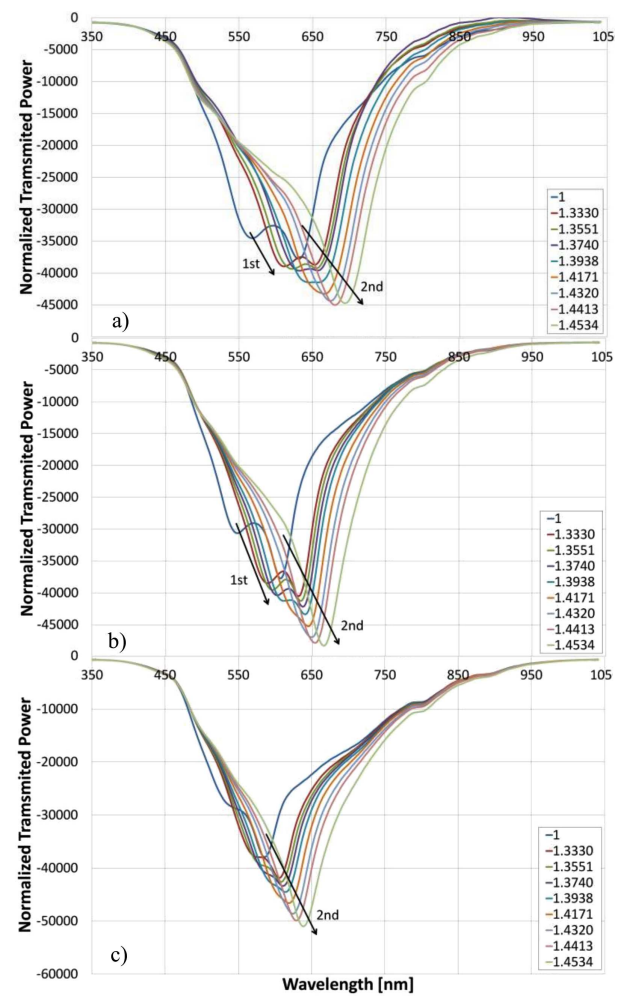

Fig. 6. Evolution of transmission spectra for sample obtained with (a) no rotation, (b) one $180^{\circ}$ rotation, and (c) three $90^{\circ}$ rotations, for external RI in range $n_{D}=1$ to 1.4534 RIU. Arrows indicate shift of the resonance with external RI.

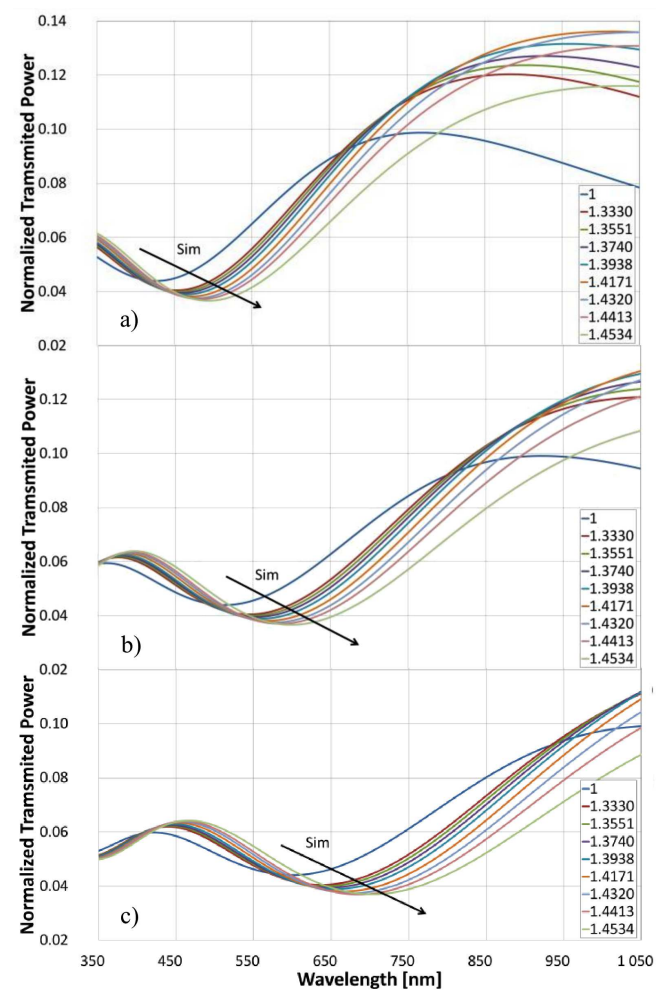

Fig. 7. Simulated evolution of transmission spectra for overlay thickness of (a) 100, (b) 120, and (c) $140 \mathrm{~nm}$. Arrows indicate shift of the resonance with external RI.

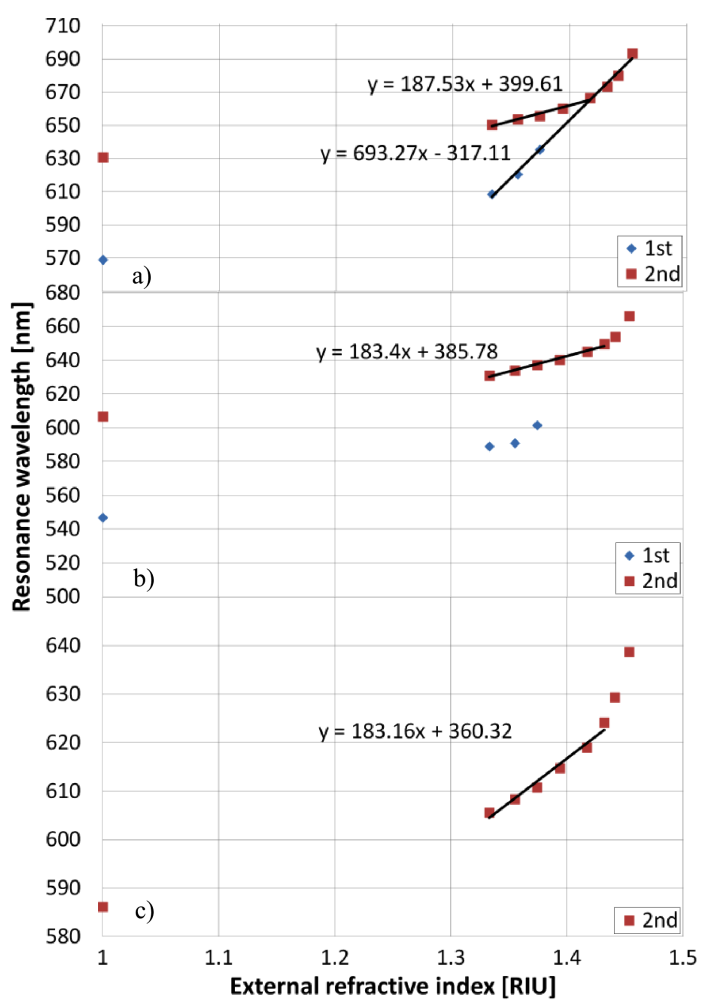

Fig. 8. Shift of the resonance wavelength with external RI for sample where (a) no rotation, (b) one $180^{\circ}$ rotation, and (c) three $90^{\circ}$ rotations between the $\mathrm{SiN}_{x}$ depositions have been performed. 
The numerical results, shown in Fig. 7 are made for idealized symmetric structure, but they carry valuable qualitative information. Firstly, they show that the perfect symmetric shape favors single resonance behavior of structure in the considered spectral range. Secondly, with the increasing thickness of the overlay the resonance wavelength shifts towards its longer values.

Comparing experimental and numerical results, it can be seen that the rotation causes an excessive resonance to decay (Fig. 6c), and thus it can be stated that rotation provides more uniform overlays. Moreover, the observed wavelength shift towards its shorter values with thinner $\mathrm{SiN}_{x}$ layer also agrees with the numerical simulations.

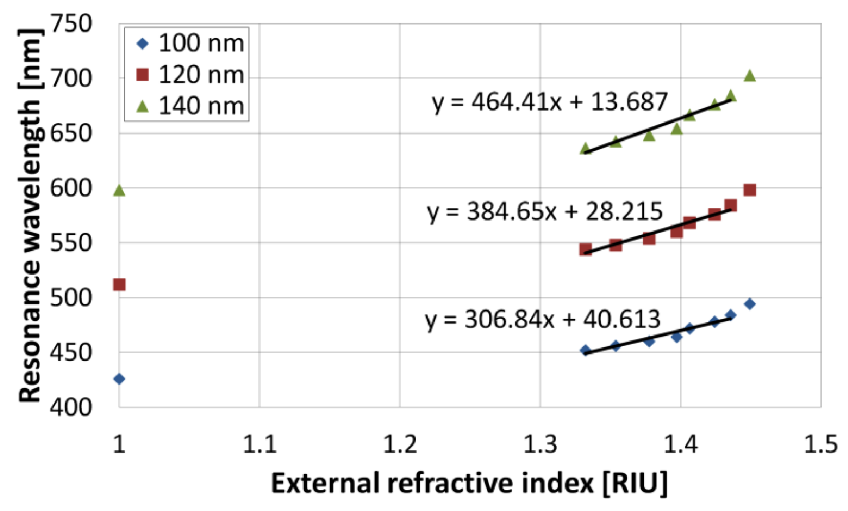

Fig. 9. Simulated shift of the resonance wavelength with external RI for simulation of overlay thickness of 100, 120, and $140 \mathrm{~nm}$.

The resonance wavelengths shift induced by external RI changes for each of the samples is shown in Fig. 8. The corresponding curves obtained on the basis of numerical calculations are shown in Fig. 9. In general, in Fig. 8 it can be seen that the rotation has no or very small impact on the sensitivity of the main resonance. With increasing RI values, the sensitivity also varies. Characteristics shown in Fig. 9 confirm the trend of sensitivity increase with thickness of deposited overlay.

\section{Conclusions}

Silicon nitride is a promising material for optical fiber sensing devices. Due to high refractive index of the $\operatorname{SiN}_{x}$, its thin overlays can modify RI sensitivity of the fiberbased devices. Rotation of the optical fiber samples suspended in plasma reactor during the RF PECVD process significantly modifies their transmission spectrum, as well as their response to external RI. Observed shift of resonances appearing at about $\lambda=600 \mathrm{~nm}$ towards shorter wavelengths is most likely induced by slight change of thickness of the deposited overlays. There is also a reduction in amount of resonances observed, which may be correlated with increases in uniformity of the overlays. Higher RI sensitivity can be achieved by deposition of thicker overlay or higher asymmetry of the coating. The sensitivity to RI obtained in this work reaches
$690 \mathrm{~nm} / \mathrm{RIU}$ and can be further optimized by proper selection of thickness, optical properties and symmetry of the overlay.

\section{Acknowledgments}

The authors gratefully acknowledge support for this work from the National Centre for Research and Development of Poland within the LIDER program.

\section{References}

[1] M.S. Meyer, G.L. Eesley, Rev. Sci. Instrum. 58, 2047 (1987).

[2] M. Smietana, J. Szmidt, M. Dudek, P. Niedzielski, Diam. Relat. Mater. 13, 954 (2004).

[3] B.J.-C. Deboux, E. Lewis, P.J. Scully, R. Edwards, J. Lightwave Technol. 13, 1407 (1995).

[4] A.P. Ferreira, M.M. Werneck, R.M. Ribeiro, Biosens. Bioelectron. 16, 399 (2001).

[5] I. Del Villar, C.R. Zamarreño, M. Hernaez, F.J. Arregui, I.R. Matias, Opt. Expr. 18, 20183 (2010).

[6] M. Smietana, W.J. Bock, J. Szmidt, Thin Solid Films 519, 6339 (2011).

[7] I. Del Villar, C.R. Zamarreño, P. Sanchez, M. Hernaez, C.F. Valdivielso, F.J. Arregui, I.R. Matias, J. Opt. 12, 095503 (2010).

[8] I. Del Villar, M. Hernaez, C.R. Zamarreño, P. Sánchez, C. Fernández-Valdivielso, F.J. Arregui, I.R. Matias, Appl. Opt. 51, 4298 (2012).

[9] M. Smietana, M. Dudek, M. Koba, B. Michalak, Phys. Status Solidi A 210, 2100 (2013). 